\title{
Pyoderma gangrenosum triggered by red tattoo dye
}

\author{
Ivan V. Litvinov MD PhD, Denis Sasseville MD
}

$\mathrm{A}$

39-year-old woman with a history of regional enteritis presented with an itchy rash on a tattoo on her left leg that she had received two weeks earlier (Figure 1A). We diagnosed allergic contact dermatitis and prescribed clobetasol propionate cream $(0.05 \%)$. About three weeks later, she returned because of a painful ulcer with undermined greyish borders that was restricted to the red-dye portion of her tattoo (Figure 1B). Results of patch testing with standard and textile series and the dyes used in her tattoo showed a nonrelevant allergy to nickel. Fungal and bacterial culture results were negative, and we ruled out systemic sarcoidosis, which may rarely present with cutaneous ulceration.

Our presumed diagnosis was pyoderma gangrenosum triggered by a pathergic reaction to allergic contact dermatitis. Although we considered severe ulcerating hypersensitivity to tattoo dye (e.g., naphthol red, a known sensitizer) as a possible diagnosis, this reaction often involves the entire tattoo surface; ${ }^{1}$ some of the red-dye portion of our patient's tattoo was not involved by the centrifugally expanding ulcer, and testing did not show a clear link. Patch testing with tattoo dyes has low sensitivity, and we opted against intradermal testing to avoid triggering another reaction. ${ }^{2}$ The ulcer resolved over four weeks (Figure 1C) with a tapering dose of oral prednisone $(1 \mathrm{mg} / \mathrm{kg})$ and topical wound care.

Pyoderma gangrenosum is a painful, ulcerative neutrophilic dermatosis seen in $1 \%-5 \%$ of patients with inflammatory bowel disease. ${ }^{3}$ The skin reaction is likely an autoimmune process, characterized by a sterile neutrophilic infiltrate. ${ }^{3,4}$ Although some cases are idiopathic, most are associated with systemic diseases such as inflammatory bowel disease, arthritis and hematologic cancers. ${ }^{4,5}$ Pyoderma gangrenosum can precede an exacerbation of inflammatory bowel disease. ${ }^{3}$ It is most commonly triggered by a pathergic response to trauma. ${ }^{4,5}$ (Pathergy can rarely develop after an allergic reaction. ${ }^{6}$ ) The diagnosis is largely clinical, based on the patient's history and the mor-
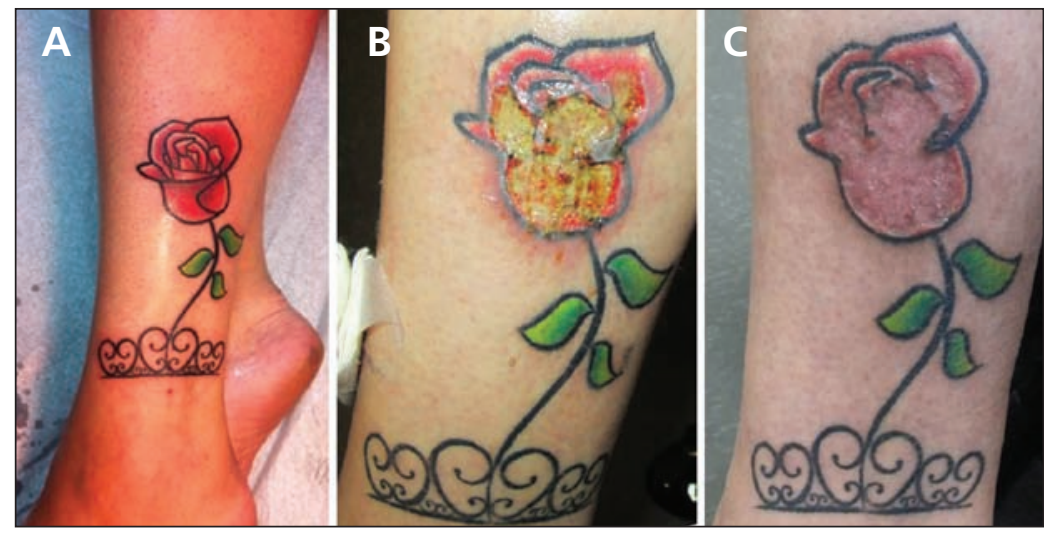

Figure 1: (A) The tattoo immediately after completion on the left lower leg of a 39-year-old woman with regional enteritis. (B) Ulcerative pyoderma gangrenosum localized to the red-dye portion of the tattoo. (C) Resolution after a fourweek course of oral prednisone.

phology of the ulcer. ${ }^{4}$ Characteristic features include preferential location on the legs, disproportionate pain, rapid ulcer extension and presence of a dusky blue or gunmetal-coloured undermined border. ${ }^{4}$ Pyoderma gangrenosum often responds well to brief immunosuppressive therapy (e.g., prednisone or cyclosporine) or, in less severe cases, to topical or intralesional corticosteroid therapy. ${ }^{4}$ People with a history of pyoderma gangrenosum or inflammatory bowel disease should be made aware of this risk before consenting to cosmetic procedures such as tattoos.

\section{References}

1. Wollina U. Severe adverse events related to tattooing: a retrospective analysis of 11 years. Indian J Dermatol 2012;57:439-43.

2. Greve B, Chytry R, Raulin C. Contact dermatitis from red tattoo pigment (quinacridone) with secondary spread. Contact Dermatitis 2003;49:265-6.

3. Agarwal A, Andrews JM. Systematic review: IBD-associated pyoderma gangrenosum in the biologic era, the response to therapy. Aliment Pharmacol Ther 2013;38:563-72.

4. Pereira N, Brites MM, Goncalo M, et al. Pyoderma gangrenosum - a review of 24 cases observed over 10 years. Int $J$ Dermatol 2013;52:938-45.

5. Jacobson S, Martin DB, Deng A, et al. Pyoderma gangrenosum following tattoo placement in a patient with acute myelogenous leukemia. J Dermatolog Treat 2008;19:58-60.

6. Lenane P, McKenna D, Murphy GM. Pyoderma gangrenosum secondary to allergic contact dermatitis from rubber. Contact Dermatitis 1998;38:238.
Competing interests: None declared.

The authors have obtained patient consent

This article has been peer reviewed

Affiliations: Division of Dermatology (Litvinov, Sasseville), McGill

University Health Centre, Montréal, Que.

Correspondence to: Denis Sasseville, denis.sasseville@mcgill.ca

CMAJ 2014. DOI:10.1503 /cmaj.140122 